\title{
Shadows and spirals in the protoplanetary disk HD 100453 ${ }^{\star}$
}

\author{
M. Benisty ${ }^{1}$, T. Stolker ${ }^{2}$, A. Pohl ${ }^{3}$, J. de Boer ${ }^{4}$, G. Lesur ${ }^{1}$, C. Dominik ${ }^{2}$, C. P. Dullemond ${ }^{5}$, M. Langlois ${ }^{6,19}$, M. Min ${ }^{7,2}$, \\ K. Wagner ${ }^{8}$, T. Henning ${ }^{3}$, A. Juhasz ${ }^{9}$, P. Pinilla ${ }^{4,8}$, S. Facchini ${ }^{10}$, D. Apai ${ }^{8}$, R. van Boekel ${ }^{3}$, A. Garufi ${ }^{11,12}$, C. Ginski $^{4}$, \\ F. Ménard ${ }^{1}$, C. Pinte ${ }^{1}$, S. P. Quanz ${ }^{12}$, A. Zurlo ${ }^{13,14,19}$, A. Boccaletti ${ }^{15}$, M. Bonnefoy ${ }^{1}$, J. L. Beuzit ${ }^{1}$, G. Chauvin ${ }^{1}$, \\ M. Cudel $^{1}$, S. Desidera ${ }^{16}$, M. Feldt ${ }^{3}$, C. Fontanive ${ }^{17}$, R. Gratton ${ }^{16}$, M. Kasper ${ }^{18,1}$, A.-M. Lagrange ${ }^{1}$, H. LeCoroller ${ }^{19}$, \\ D. Mouillet ${ }^{1}$, D. Mesa ${ }^{16}$, E. Sissa ${ }^{16}$, A. Vigan ${ }^{19}$, J. Antichi ${ }^{20}$, T. Buey ${ }^{15}$, T. Fusco ${ }^{21,19}$, D. Gisler ${ }^{12}$, M. Llored ${ }^{19}$, \\ Y. Magnard ${ }^{1}$, O. Moeller-Nilsson ${ }^{3}$, J. Pragt ${ }^{2}$, R. Roelfsema ${ }^{2}$, J.-F. Sauvage ${ }^{21,19}$, and F. Wildi ${ }^{22}$
}

(Affiliations can be found after the references)

Received 27 September 2016 / Accepted 28 October 2016

\begin{abstract}
Context. Understanding the diversity of planets requires studying the morphology and physical conditions in the protoplanetary disks in which they form.

Aims. We aim to study the structure of the $\sim 10$ Myr old protoplanetary disk HD 100453, to detect features that can trace disk evolution and to understand the mechanisms that drive these features.

Methods. We observed HD 100453 in polarized scattered light with VLT/SPHERE at optical $(0.6 \mu \mathrm{m}, 0.8 \mu \mathrm{m})$ and near-infrared (1.2 $\mu \mathrm{m})$ wavelengths, reaching an angular resolution of $\sim 0.02^{\prime \prime}$, and an inner working angle of $\sim 0.09^{\prime \prime}$.

Results. We spatially resolve the disk around HD 100453, and detect polarized scattered light up to $\sim 0.42^{\prime \prime}$ ( $\sim 48$ au). We detect a cavity, a rim with azimuthal brightness variations at an inclination of $\sim 38^{\circ}$ with respect to our line of sight, two shadows and two symmetric spiral arms. The spiral arms originate near the location of the shadows, close to the semi major axis. We detect a faint feature in the SW that can be interpreted as the scattering surface of the bottom side of the disk, if the disk is tidally truncated by the M-dwarf companion currently seen at a projected distance of $\sim 119 \mathrm{au}$. We construct a radiative transfer model that accounts for the main characteristics of the features with an inner and outer disk misaligned by $\sim 72^{\circ}$. The azimuthal brightness variations along the rim are well reproduced with the scattering phase function of the model. While spirals can be triggered by the tidal interaction with the companion, the close proximity of the spirals to the shadows suggests that the shadows could also play a role. The change in stellar illumination along the rim induces an azimuthal variation of the scale height that can contribute to the brightness variations.

Conclusions. Dark regions in polarized images of transition disks are now detected in a handful of disks and often interpreted as shadows due to a misaligned inner disk. However, the origin of such a misalignment in HD 100453, and of the spirals, is still unclear, and might be due to a yet-undetected massive companion inside the cavity, and on an inclined orbit. Observations over a few years will allow us to measure the spiral pattern speed, and determine if the shadows are fixed or moving, which may constrain their origin.
\end{abstract}

Key words. protoplanetary disks - radiative transfer - techniques: polarimetric

\section{Introduction}

Thousands of exoplanetary systems have been detected so far displaying a wide diversity in their architecture. Understanding planet formation and its outcomes requires good knowledge of the protoplanetary disks at different spatial scales. Although forming planets have not been unambiguously detected so far, one can aim to study the conditions for their formation by looking for indirect signatures and imprints of the mechanisms driving the disk evolution.

In recent years, high resolution images of protoplanetary disks have shown a variety of small-scale features. In the submillimeter regime, one of the most stunning images was obtained using ALMA at its highest angular resolution, on HL Tau, a very young object $(0.5$ Myrold, ALMA Partnership et al. 2015), and revealed concentric rings in a very flat disk (Carrasco-González et al. 2016; Pinte et al. 2016). These rings indicated that planet formation might occur very early in the disk lifetime, but alternative explanations, such as hydrodynamical instabilities were also proposed (Flock et al. 2015; Ruge et al. 2016; Béthune et al. 2016). Interestingly, rings were also detected in the sub-millimeter observations of a very old disk,

\footnotetext{
^ Based on observations performed with VLT/SPHERE under program ID 096.C-0248(B)
}

TW Hya (10 Myr old; Andrews et al. 2016; Tsukagoshi et al. 2016) suggesting that such small features are ubiquitous and/or long lived. On the other hand, other sub-millimeter images showed, azimuthally asymmetric brightness enhancements in continuum (Casassus et al. 2012; van der Marel et al. 2015; Pérez et al. 2014; Pinilla et al. 2015c) and in very few objects, spiral arms (Christiaens et al. 2014; Pérez et al. 2016). The diversity of these features supports the idea that several processes (e.g., planet formation, hydrodynamical instabilities, photoevaporation) might act simultaneously and with different relative contribution depending on the object.

Stunning images of the scattering surfaces of protoplanetary disks are produced with polarimetric differential imaging (PDI; e.g. Kuhn et al. 2001; Apai et al. 2004; Quanz et al. 2011). The technique consists of measuring the linear polarization of the light scattered by dust grains in the disk and to remove the unpolarized contribution, including that from the star. Recent images show rings (e.g. Rapson et al. 2015; Wolff et al. 2016; Ginski et al. 2016), spiral arms (e.g., Muto et al. 2012; Grady et al. 2013; Benisty et al. 2015; Stolker et al. 2016a), localized dips (e.g., Pinilla et al. 2015a; Canovas et al. 2016) and shadows (e.g., Avenhaus et al. 2014). As these observations only trace small dust grains in the upper disk layers, and not the bulk of the disk mass, these features may trace enhancements 
in surface density, or variations in the disk scale height due to local heating events (Juhász et al. 2015; Pohl et al. 2015). These features have now been observed in disks surrounding stars with a broad range of properties in terms of stellar luminosity, age and disk evolution.

Of particular interest for this paper is HD 100453 A, hereafter referred to as simply HD 100453, a Herbig A9Ve star located in the Lower Centaurus Association (Kouwenhoven et al. 2005), at $\sim 114_{-4}^{+11}$ pc (Perryman et al. 1997), with an early-M star companion (Chen et al. 2006). In a detailed multi-wavelength study, Collins et al. (2009) refined the age of the system to be $10 \pm 2 \mathrm{Myr}$, and also constrained the companion properties. It is an M4.0-M4.5V, $0.20 \pm 0.04 M_{\odot}$ star, located at $1.045^{\prime \prime} \pm 0.025^{\prime \prime}$ (i.e., $\sim 119 \mathrm{au}$ ) at a PA of $126 \pm 1^{\circ}$. HD 100453 was classified as a Group I (flared) disk by Meeus et al. (2001). The disk reprocesses a significant fraction of the stellar light in the inner and outer disk regions suggesting a vertically thick and flared disk (Dominik et al. 2003). Interestingly, there is no clear sign of accretion onto the star. Collins et al. (2009) derived an accretion rate upper limit of $1.4 \times 10^{-9} M_{\odot} / \mathrm{yr}$ from the far-UV continuum, confirmed by Fairlamb et al. (2015; upper limit of $\left.4.9 \times 10^{-9} M_{\odot} / \mathrm{yr}\right)$. HD 100453 gas tracers also show a peculiarity: while Herbig stars with a strong near-infrared (NIR) excess show $4.7 \mu \mathrm{m} \mathrm{CO}$ emission (Brittain et al. 2007), HD 100453 does not show any (Collins et al. 2009), which suggests a high dust-to-gas ratio or a reduction of the gas content in the inner disk. Collins et al. (2009) report a non-detection of CO $J=3-2$ with the JCMT, that indicates that the gas amount in the outer disk region might also be severely reduced. From the $1.2 \mathrm{~mm}$ continuum emission, and the $\mathrm{CO}$ upper limit, the disk mass is estimated to be $8 \times 10^{-5} M_{\odot}$, and the gas to dust ratio to be not more than 4:1 (Collins et al. 2009).

The disk surrounding HD 100453 must be relatively compact, compared to other Herbig Ae disks. HST observations report no scattered light detection beyond $3^{\prime \prime}$ (Collins et al. 2009). A background star is detected at a projected distance of $90 \mathrm{au}$, which indicates that the disk is either truncated by tidal interaction with the M-dwarf companion, or optically thin, at this projected distance from the star. This is supported by two marginally resolved images, at $\sim 0.2^{\prime \prime}-0.3^{\prime \prime}$ scales (i.e., $\sim 25-35 \mathrm{au}$ ), in the PAH and $Q$-band filters (Habart et al. 2006; Khalafinejad et al. 2016). Using SPHERE with differential imaging, Wagner et al. (2015a) reported the detection of two spiral arms in scattered light, up to $0.37^{\prime \prime}(\sim 42 \mathrm{au})$, and a marginal detection of a gap or cavity inside $0.18^{\prime \prime}(\sim 20 \mathrm{au})$.

In this paper, we report the first polarized differential images of HD 100453 obtained in the optical ( $R^{\prime}$ and $I^{\prime}$ bands) and in the near infrared ( $J$ band) with VLT/SPHERE. The paper is organized as follows. Section 2 describes the observations and the data processing. Section 3 reports on the detected disk features, Sect. 4 provides a radiative transfer model that well reproduces the observations and in Sect. 5 we discuss our findings.

\section{Observations and data reduction}

The observations were carried out on March 30th and 31th, 2016, with the SPHERE instrument (Beuzit et al. 2008; and in prep.), equipped with an extreme adaptive-optics (AO) system (Fusco et al. 2006; Petit et al. 2014; Sauvage et al. 2014) at the Very Large Telescope at Cerro Paranal, Chile. The observations were executed through the Guaranteed Time program. HD 100453 was observed in the $R^{\prime}$ and $I^{\prime}$ band filters $\left(\lambda_{0}=0.626, \Delta \lambda=0.149 \mu \mathrm{m} ; \lambda_{0}=0.790, \Delta \lambda=0.153 \mu \mathrm{m}\right.$, respectively) using the ZIMPOL instrument (Roelfsema et al. 2010; Thalmann et al. 2008) with a plate scale of 3.5 milliarcseconds (mas) per pixel and in the $J$ band $\left(\lambda_{0}=1.258\right.$, $\Delta \lambda=0.197 \mu \mathrm{m})$ using the infrared dual-band imager and spectrograph (IRDIS; Dohlen et al. 2008; Langlois et al. 2014), with a plate scale of 12.25 mas per pixel.

We used a 185 mas-diameter coronographic focal mask combined with an apodized pupil and Lyot stop. HD 100453 was observed for 85 and 80 min with IRDIS and ZIMPOL, respectively. These data were taken under moderate AO conditions (seeing between 0.7 and $1.0^{\prime \prime}$ ). From an analysis of the reference point spread function (PSF), we find that the AO quality reaches a diffraction-limited regime, with a $20.8 \times 24$ mas resolution (slightly elongated PSF due to wind speed, the theoretical diffraction limit being 20.6 mas) and a $43 \%$ Strehl Ratio at $0.8 \mu \mathrm{m}$.

For polarimetric differential imaging, the instruments split the beam into two orthogonal polarization states. The half-wave plate (HWP) that controls the orientation of the polarization, and allows to decrease the effect of instrumental polarization, was set to four positions shifted by $22.5^{\circ}$ in order to construct a set of linear Stokes vectors. The data was reduced according to the double difference method (Kuhn et al. 2001), which is described in detail for the polarimetric modes of IRDIS and ZIMPOL in de Boer et al. (2016), and lead to the Stokes parameters $Q$ and $U$. Under the assumption of single scattering, the scattered light from a circumstellar disk is expected to be linearly polarized in the azimuthal direction. Hence, we describe the polarization vector field in polar rather than Cartesian coordinates (Avenhaus et al. 2014) and define the polar-coordinate Stokes parameters $Q_{\Phi}$ and $U_{\Phi}$ as:

$Q_{\Phi}=+Q \cos (2 \Phi)+U \sin (2 \Phi)$,

and

$U_{\Phi}=-Q \sin (2 \Phi)+U \cos (2 \Phi)$,

where $\Phi$ is the position angle of the location of interest $(x, y)$ with respect to the star location $\left(x_{0}, y_{0}\right)$, and is written as:

$\Phi=\arctan \frac{x-x_{0}}{y-y_{0}}+\theta$

$\theta$ corrects for instrumental effects such as the angular misalignment of the HWP. In this coordinate system, the azimuthally polarized flux from a circumstellar disk appears as a consistently positive signal in the $Q_{\phi}$ image, whereas the $U_{\phi}$ image remains free of disk signal and provides a convenient estimate of the residual noise in the $Q_{\phi}$ image (Schmid et al. 2006). To determine the absolute disk surface brightness in polarized intensity requires advanced calibration of the polarimetric throughput of the system, which lies beyond the scope of this study. We therefore use arbitrary units in the images shown in the paper.

In Fig. 1, we present the resulting polarized scattered light images in the optical and NIR. We note that there is a residual signal in the $U_{\phi}$ image, in particular in the $R^{\prime}$ band image, that may be due to multiple scattering events (Canovas et al. 2015).

\section{Polarized intensity images}

The images of Fig. 1 reveal a number of disk features. The NIR image shows the same features as the optical ones, albeit with a lower angular resolution, leading to fuzzier features. 

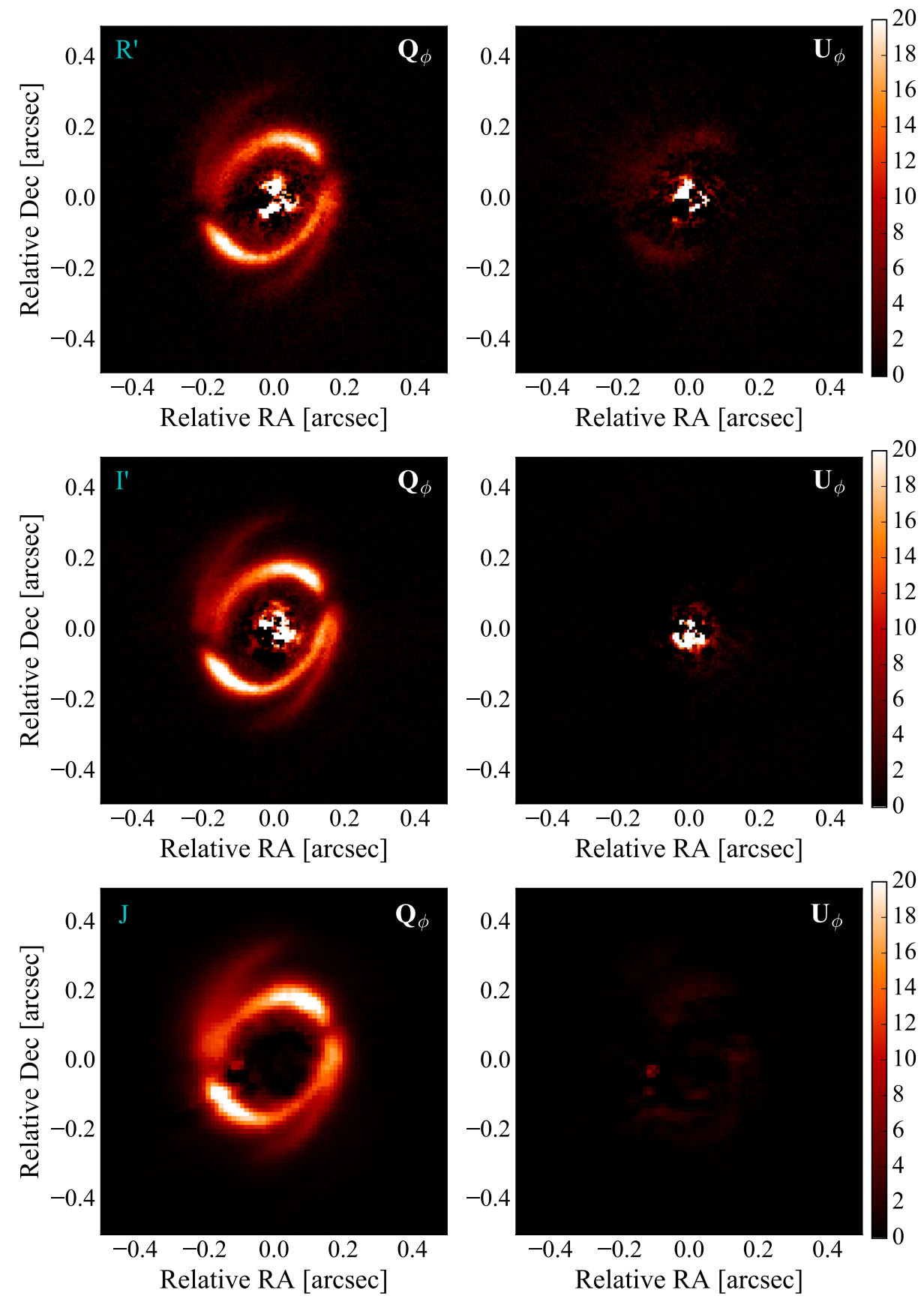

Fig. 1. $R^{\prime}($ top $), I^{\prime}$ (middle), and $J$ band (bottom) polarized intensity images, $Q_{\phi}(l e f t)$ and $U_{\phi}(r i g h t)$. In the optical images, the inner bright region corresponds to saturated pixels inside our IWA. In the NIR images, the inner dark region is masked by the coronagraph. The color scale of the $Q_{\phi}$ and $U_{\phi}$ are the same, and arbitrary. For all images, East is pointing left.

Looking at the optical images (Fig.1, top and middle), beyond a distance of $0.09^{\prime \prime}(\sim 10 \mathrm{au})$ that corresponds to the inner working angle (IWA) of our observations, we detect, from inside out:

(a) a region with low scattered light signal, called cavity, from our IWA up to $\sim 0.14^{\prime \prime}(\sim 16 \mathrm{au})$. We note that although we can not probe inside $0.09^{\prime \prime}$, the NIR excess seen in the spectral energy distribution (SED) indicates the presence of a significant amount of dust grains in the inner au(s). The inner working angle therefore provides an upper limit on the outer radius of the inner disk.

(b) a ring-like feature, called the $\mathrm{rim}$, located at $\sim 0.14^{\prime \prime}(\sim 16 \mathrm{au})$ with an apparent width ranging from $\sim 0.050$ to $\sim 0.075^{\prime \prime}(\sim 5$ to $9 \mathrm{au}$ ). Its brightness varies azimuthally, and there are two clear maxima at PAs $\sim 135^{\circ}$ and $\sim 325^{\circ}$. The brightest regions are distributed over an azimuthal range of $\sim 70^{\circ}$.

(c) two dark regions along the rim, that we refer to as shadows. These regions are located at $\sim 100^{\circ}$ and $\sim 293^{\circ}$ and have an angular extent of $\sim 12^{\circ}$ at the inner edge of the rim, that slightly increases with radius.

(d) two spiral arms, in the NE and the SW, extending to $\sim 0.42^{\prime \prime}$ ( $\sim 8 \mathrm{au})$ and $\sim 0.34^{\prime \prime}$ ( $\left.\sim 39 \mathrm{au}\right)$, respectively. Interestingly, the spirals are located very close to the shadows.

(e) an additional spiral-like feature, in the SW. This feature can be seen in Fig. 2, in which we scale the $J$-band image by $r^{2}$ to compensate for the $r^{-2}$ dependency of the stellar illumination, and enhance faint features located further out in the disk. 


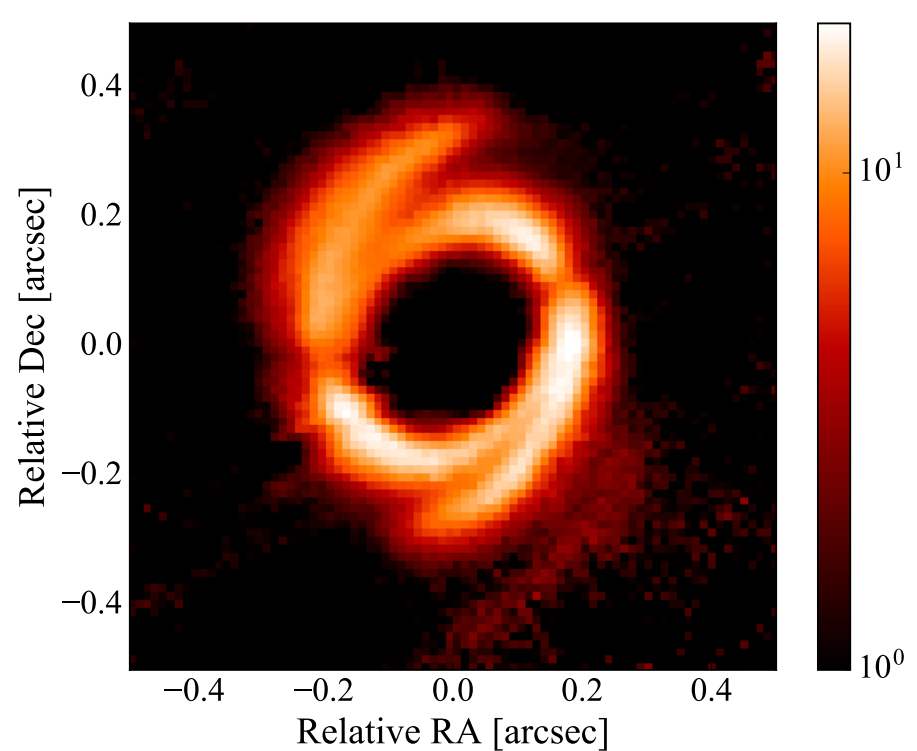

Fig. 2. $J$-band $Q_{\phi}$ image after scaling each pixel by the square of its distance from the star, $r^{2}$. The scaling takes into account the geometry of the $\tau=1$ surface given by our radiative transfer model. The color log-scale is arbitrary.

The values of $r^{2}$ applied to the original image take into account the inclination and PA of the object, as well as the disk flaring following the method described by Stolker et al. (2016b; and using a $\tau=1$ surface with $h=0.22 \times r^{1.04}$ with $r$ and $h$ in au, as derived from our radiative transfer model, see Sect. 4). This feature is also detected in the ZIMPOL data, in the differential imaging data by Wagner et al. (2015a) and in newly acquired angular differential images with SPHERE (see Fig. A.1).

In all images, the NE spiral appears to have a larger opening angle than the SW spiral. If we assume that the disk is inclined and flared, and that the spirals intrinsic opening angles are similar, this may indicate that the NE is the far side of the disk and the SW its near side. This is supported by the smaller width of the rim in the SW and of the shadow in the West, and by the fact that the SW spiral is twice as bright as the NE spiral in the total intensity images of Wagner et al. (2015a), assuming that this effect is due to forward-scattering. Finally, if the disk is truncated by the M-dwarf, the faint additional spiral-like feature in the SW may be tracing scattered light from the outer edge of the bottom side of the disk. Assuming that the images in Fig. 1 show signal from the disk surface layer at a given height $\sim h$ from the disk midplane, this additional spiral-like feature would trace the layer at $\sim-h$, on the other side of the disk midplane. This scenario would support the idea that the SW is indeed the near side of the disk.

To determine the inclination and the position angle of the rim, we fit an ellipse to the brightest point along each radius in the optical image, and find major and minor axes corresponding to an inclination of $\sim 38^{\circ}$, in close agreement with the value found by Wagner et al. $\left(2015 \mathrm{a} ; 34^{\circ}\right)$. A position angle of $\sim 142^{\circ}$ and a shift of the ellipse center by $\sim 7$ mas in the SE fit the data best. This offset could originate from either the vertical thickness of the inclined rim (assumed to be zero in our 2D-ellipse fitting), a non-zero eccentricity of the rim, an effect of the dust grain scattering phase function, or a combination of some or all of these.

In Fig. 3 we present the polar mapping of the $I^{\prime}$ image, after deprojection using $i=38^{\circ}$ and $\mathrm{PA}=142^{\circ}$. It clearly shows the shadows and the azimuthal brightness variations. Interestingly,

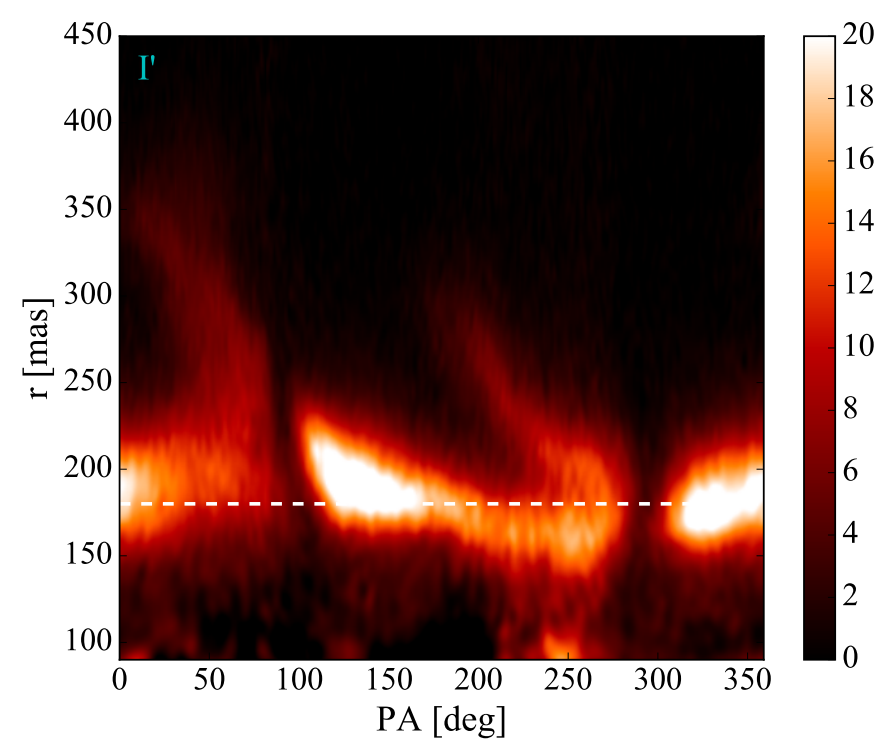

Fig. 3. Polar map of the deprojected $I^{\prime} Q_{\phi}$ image using $i=38^{\circ}$ and $\mathrm{PA}=142^{\circ}$. The dashed line indicates a radius of $0.18^{\prime \prime}$. The color scale is arbitrary.

the NE spiral seems to appear on both sides of the shadow (at approximatively $\mathrm{PA} \sim 100^{\circ}$ ).

Figure 4 shows the radial and azimuthal cuts, when averaging azimuthally and across the rim width $\left(0.17^{\prime \prime}-0.20^{\prime \prime}\right)$, respectively. The error bars are estimated as the standard deviation in each bin in the $U_{\phi}$ image. We measure a ratio of (radially averaged) polarized surface brightness of $\sim 5$ between the shadows and the brightest regions of the rim.

\section{Radiative transfer modeling}

In this section, we aim to provide a radiative transfer model for HD 100453, that reproduces the main characteristics of the rim, the spirals and the shadows seen in the scattered light images, in particular, their locations, widths, and brightness variations.

\subsection{MCMax3D model}

We use the 3D version of the continuum radiative transfer code MCMax (Min et al. 2009) which calculates the thermal structure of the disk and produces ray-traced images. We consider an inner disk, a cavity and an outer disk. The dust surface density is parametrized radially as:

$\Sigma(r) \propto r^{-\epsilon} \exp \left[-\left(\frac{r}{R_{\text {tap }}}\right)^{2-\epsilon}\right]$,

where $R_{\text {in }}<r<R_{\text {out }}$ is the disk radius, $R_{\text {tap }}$ the tapering-off radius and $\epsilon$ the surface density power law index. The surface density profile is scaled to the total dust mass, $M_{\text {dust }}$ and the vertical density distribution follows a Gaussian profile. The disk aspect ratio is parametrized radially as $H(r) / r=\left(H_{0} / r_{0}\right)\left(r / r_{0}\right)^{\psi}$ with $H(r)$ being the scale height, $H_{0} / r_{0}$ the aspect ratio at the reference radius $r_{0}$, and $\psi$ the flaring index.

We consider a minimum $\left(a_{\min }\right)$ and maximum $\left(a_{\max }\right)$ grain size and use a power law for the dust grain size distribution with an index $\gamma$. We use a dust mixture made of $70 \%$ silicates and $30 \%$ carbon (DIANA, Woitke et al. 2016), and the porosity of the grains is set to $25 \%$. We consider the grains to be irregular in shape by setting the maximum volume void fraction used for the 

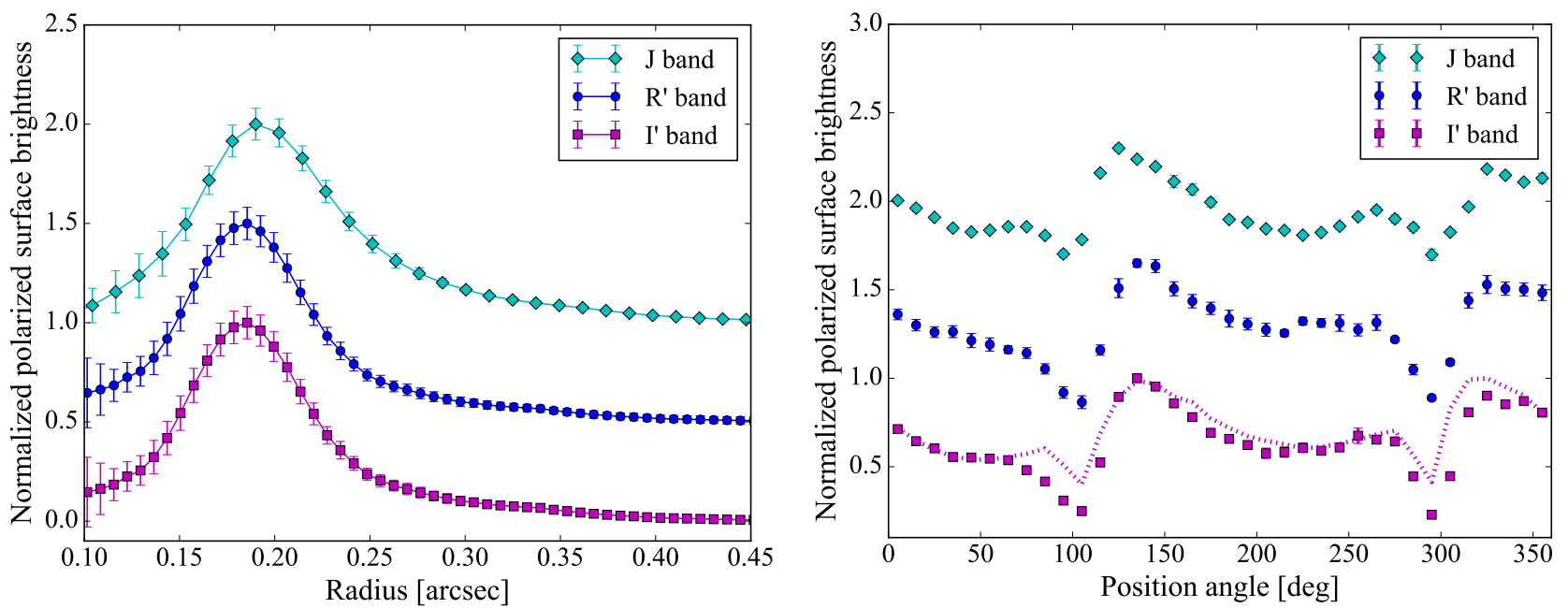

Fig. 4. Left: normalized radial cuts of the $R^{\prime}, I^{\prime}$ and $J$-band images after averaging azimuthally. Right: normalized azimuthal cuts of the $R^{\prime}, I^{\prime}$ and $J$-band images, after averaging radially between 170 and 200 mas. The radiative transfer model prediction (dotted curve) reproduces the observed azimuthal brightness variations relatively well. Note that due to the large variation of surface brightness along the rim, the standard deviation in each bin can vary from 2 to $17 \%$. The curves are shifted vertically for clarity.

distribution of hollow spheres (DHS) method to 0.8 (Min et al. 2005).

We describe the spiral arms as Archimedean spirals, following $r(\theta)=A 1+A 2 *\left(\theta-\theta_{0}\right)^{n}$. We assume that they trace perturbations in the disk scale height, rather than in the surface density. Hence, along the spirals, the scale height is multiplied by $1+a_{\text {height }} * \exp ^{((r-r(\theta)) / w)^{2}} *(A 1 / r)^{q}$, where $\mathrm{w}$ is the width of the spiral and $q$ determines the steepness of the radial falloff of the spiral arm.

Once the temperature structure is computed, synthetic SEDs and ray-traced polarized images can be produced at any wavelength. We compute monochromatic Stokes $Q_{\phi}$ and $U_{\phi}$ images, at $0.79 \mu \mathrm{m}$, and use an unsaturated observed Stokes $I$ frame as a PSF to convolve the synthetic maps.

\subsection{Best model}

We generate the shadows using a misaligned inner disk, with respect to the outer disk. For the outer disk, we use the inclination and position angles derived from the ellipse fitting $\left(i \sim 38^{\circ}, \mathrm{PA} \sim 142^{\circ}\right.$; see Sect. 3), while for the inner disk, we use $i \sim 48^{\circ}$, PA $\sim 80^{\circ}$, which are obtained from geometrical model fitting of NIR interferometric observations (PIONIER survey, Lazareff et al. 2017). As the inner and outer disks must be significantly misaligned to create deep shadows (Marino et al. 2015), we assume that the near side of the outer disk is in the SW, while the near side of the inner disk is in the NE. This leads to a misalignment of $\sim 72^{\circ}$, obtained by calculating the angle between the normal vectors to the inner and outer disks. The location of the shadows depends on the orientation of the inner disk (for a given outer disk orientation), while their shape depends on the inner disk aspect ratio (the larger the aspect ratio, the broader the shadows), and on the width and roundness of the outer disk rim.

Our model parameters are summarized in Table 1. We fix the inner disk rim at 0.27 au (Klarmann et al. 2016) and its outer radius at $1 \mathrm{au}$ (Menu et al. 2015). The outer disk starts at $20 \mathrm{au}$ with a strongly peaked surface density profile (see Fig. B.1, left). We use a minimum grain size of $0.01 \mu \mathrm{m}$ and a maximum size of $1 \mu \mathrm{m}$, as larger grains result in a strong brightness asymmetry between the near and far side of the disk, due to forward scattering, which we do not observe. The outer disk mass, aspect ratio and flaring were chosen to fit the mid-IR and far-IR excesses in the SED. We note that the grain size distribution that we consider is valid for the surface layers probed in the scattered light images, but probably not valid for the disk midplane that likely hosts larger grains.

Figure 5 shows our best model that well reproduces the location of the rim and its azimuthal brightness variation as well as the width and location of the shadows (Fig. 4). The brightness contrast between the rim and the spirals is also well reproduced (Fig. 6). We find opening angles of $\sim 15^{\circ}$ at the onset of the spirals. The surface brightness is maximal on both sides of the major axis due to the high degree of polarization for $90^{\circ}$ scattering angles. A third spiral-like feature in the SW is also predicted by our model, and overlaps with the one detected in the observations, supporting the idea that it could trace the scattering surface of the bottom side of the disk, if it is truncated at $\sim 50 \mathrm{au}$ (Fig. 5). The SED is well reproduced for wavelengths longer than 10 microns, most of which is probing the outer disk, but the model misses significant emission in the NIR (Fig. B.1, right). It is a general problem that disk models fail to reproduce the NIR excess of Herbig Ae stars (e.g., Benisty et al. 2010; Flock et al. 2016; Klarmann et al. 2016), and solving this is beyond the scope of this paper. In the particular case of HD 100453, Khalafinejad et al. (2016) added an optically thin halo to reprocess a significant fraction of the stellar light. Optically thin material at high altitude was similarly considered in models of other Herbig Ae stars to reproduce the large NIR excess (e.g., Verhoeff et al. 2011; Wagner et al. 2015b).

\section{Discussion}

\subsection{Origin of the spirals}

Until now, spiral arms have been unambiguously detected in PDI observations of six Herbig Ae disks: HD 100453 (Wagner et al. 2015a, this work); AB Aur (Hashimoto et al. 2011); HD 142527 (Canovas et al. 2013; Avenhaus et al. 2014); SAO 206462 (Muto et al. 2012; Garufi et al. 2013; Stolker et al. 2016a); MWC 758 (Grady et al. 2013; Benisty et al. 2015), and HD 100546 (Ardila et al. 2007; Garufi et al. 2016). In half of 


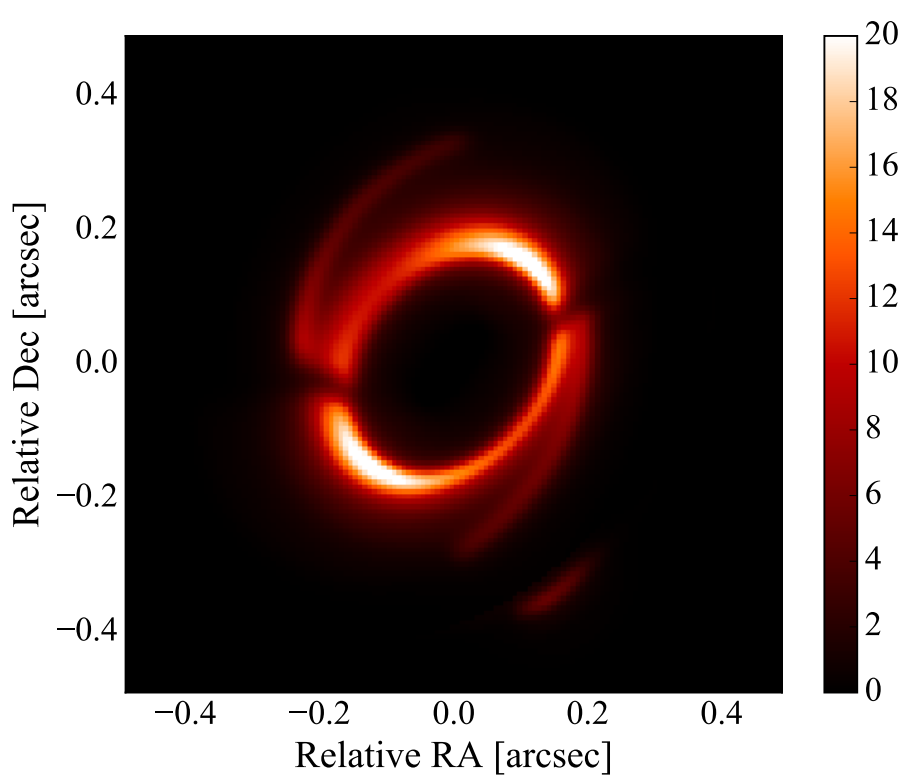

Fig. 5. Synthetic $Q_{\phi} I^{\prime}$-band polarized image from our radiative transfer model. To show it more clearly, the faint feature in the SW is enhanced by a factor of five, and traces the scattering surface of the bottom side of the disk.

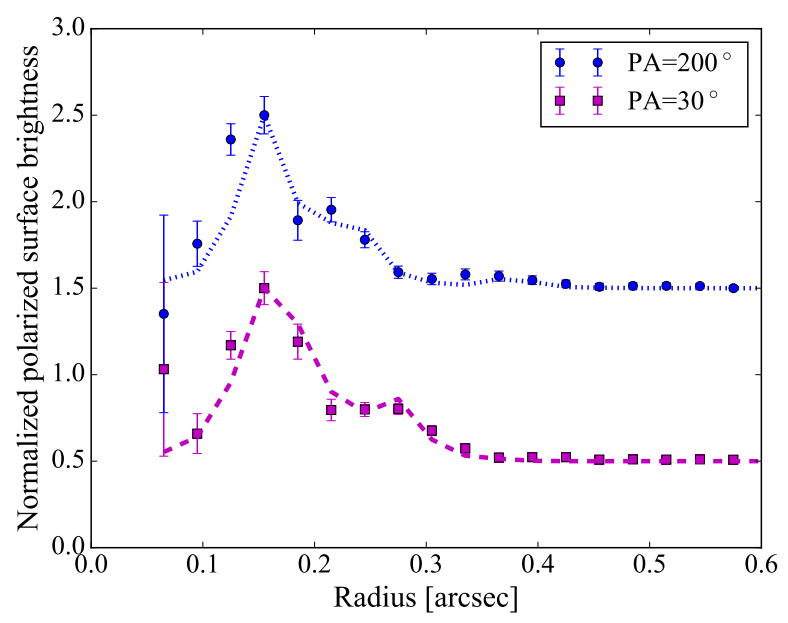

Fig. 6. Normalized radial cuts along position angles of $30^{\circ}$ and $200^{\circ}$ (obtained within a $10^{\circ}$ bin), and the corresponding model predictions (dashed and dotted lines, respectively). The curves are shifted vertically for clarity.

them, the spiral arms show an $m=2$ symmetry. Since these spirals appear in polarized scattered light, they only trace the small dust grains, well coupled to the gas, but located at the surface layers of the disks. It is difficult to know whether they originate in perturbations in the surface layers only, or if they also trace perturbations deeper in the disk. In the sub-millimeter wavelength range, that traces the bulk material of the disk, so far only two of these disks show clear spiral arms in the CO lines (Christiaens et al. 2014; Tang et al. 2012), and only one other in the continuum (Pérez et al. 2016).

Various mechanisms have been suggested for the origin of the spirals observed in disks. Planet disk interactions launch spiral waves at the Lindblad resonances (e.g. Ogilvie \& Lubow 2002), with small pitch angles, while gravitational instabilities lead to large-scale spiral arms with larger pitch angles (e.g., Lodato \& Rice 2004; Pohl et al. 2015), capable of trapping dust particles (Dipierro et al. 2015). Non-ideal
Table 1. MCMax3D model parameters.

\begin{tabular}{|c|c|c|}
\hline Parameter & Inner disk & Outer disk \\
\hline$R_{\text {in }}[\mathrm{au}]$ & 0.27 & 20 \\
\hline$R_{\text {out }}[\mathrm{au}]$ & 1 & 45 \\
\hline$R_{\mathrm{tap}}[\mathrm{au}]$ & 50 & 50 \\
\hline$M_{\text {dust }}\left[M_{\odot}\right]$ & $1 \times 10^{-10}$ & $2 \times 10^{-5}$ \\
\hline \multirow[t]{2}{*}{$\epsilon$} & 1 & -3 (rim) \\
\hline & & $1(r \geq 25 \mathrm{au})$ \\
\hline$H_{0} / r_{0}$ & 0.04 & 0.05 \\
\hline$r_{0}[\mathrm{au}]$ & 1 & 20 \\
\hline$\psi$ & 0 & 0.13 \\
\hline$\alpha$ & $10^{-3}$ & $10^{-3}$ \\
\hline$a_{\min }[\mu \mathrm{m}]$ & 0.01 & 0.01 \\
\hline$a_{\max }[\mu \mathrm{m}]$ & 1 & 1 \\
\hline$\gamma$ & -3.5 & -3.5 \\
\hline$i[\mathrm{deg}]$ & 48 & -38 \\
\hline PA [deg] & 80 & 142 \\
\hline Parameter & NE spiral & SW spiral \\
\hline$R_{\text {in }}[\mathrm{au}]$ & 27 & 38 \\
\hline$R_{\text {out }}[\mathrm{au}]$ & 33 & 45 \\
\hline $\mathrm{A} 1[\mathrm{au}]$ & 27 & 30 \\
\hline A2 [au] & 7 & 8 \\
\hline$\theta_{0}[\mathrm{deg}]$ & 125 & 125 \\
\hline$n$ & 1.12 & 1.12 \\
\hline$a_{\text {height }}$ & 0.8 & 1.1 \\
\hline$w[\mathrm{au}]$ & 1.2 & 1.2 \\
\hline$q$ & 1.7 & 1.7 \\
\hline
\end{tabular}

Notes. For the star, we used the following parameters: $T_{\text {eff }}=7400 \mathrm{~K}$, $L=8 L_{\odot}, R=1.73 R_{\odot}, M=1.66 M_{\odot}$. Note that we use a negative value for the outer disk inclination to account for the fact that the near side is in the SW.

magnetohydrodynamics (e.g., Lyra et al. 2015) and shadows can also induce spirals (Montesinos et al. 2016). While all these processes can possibly act together, gravitational instabilities are unlikely to occur in HD 100453, considering the low gas content of the disk (Collins et al. 2009) The striking symmetry of the two spiral arms seen in HD 100453 could be induced by two (yet-undetected) planets located inside the cavity. However, in this scenario, the planets should be located at symmetrical locations inside the cavity, in an unstable configuration. We find this scenario unlikely, also because the $m=2$ mode is seen in other objects.

The two symmetric spiral arms seen in HD 100453 can be induced by the tidal interaction with the low-mass companion located at a projected distance of $\sim 119$ au (Dong et al. 2016). We note, however, that to be similar to the observations, the disk model presented in Dong et al. (2016) is required to be close to face-on, which is not supported by our observations. As there is possibly a wide range of disk and orbital parameters that would likely lead to a good agreement with the observations, we cannot rule out this possibility as the origin of the spirals, and still find this scenario likely.

If not coincidental, the proximity of both of the spirals to the shadows in the polarized intensity images of HD 100453 suggests that the shadows could also play a role, and that the 
spirals might be induced by the pressure decrease at the shadows' locations (Montesinos et al. 2016; Casassus 2016). We note however, that the stellar and disk parameters considered in the hydrodynamical simulations of Montesinos et al. (2016) are very far from the ones measured for HD 100453. In particular, the Toomre parameter values for HD 100453 are much higher than the minimum ones (ranging from 0.5 to 3.4 ) in their simulations, and while it is not clear whether it is relevant for HD 100453, self-gravity might play an important role in triggering and maintaining the spirals. This is suggested by the nonstationarity of the spirals, in contrast with the expectations in the case of a steady shadow. Dedicated hydrodynamical simulations are needed to determine the conditions in which shadow-induced spirals could appear in HD 100453.

If spirals can be induced by steady shadows, the cooling timescale is required to be much shorter than the dynamical timescale ( instantaneous), otherwise the gas does not have time to adjust and the pressure gradient is not significant enough to trigger spirals. On the other hand, if the inner disk (that we assume is responsible for the shadows) precesses, the shadows are not fixed anymore. At the radius that co-rotates with the shadows, the shadowed gas is maintained in a cold region and the disk undergoes the strongest heating/cooling which might lead to spiral density waves, even with non-instantaneous cooling. For this to apply to HD 100453, the precession timescale must equal the orbital timescale at the radius where the spirals originate. We note that at the rim location $(\sim 25 \mathrm{au})$, the orbital period is $\sim 100 \mathrm{yr}$, already relatively fast compared to precession timescales (Papaloizou \& Terquem 1995).

Interestingly, the spirals generated by fixed or moving shadows are different. As in the case of a perturbing planet that corotates with the disk, if the shadows move at the precession rate of the inner disk, the spirals are trailing, and the rotational direction of the disk is counterclockwise. In contrast, if the spirals are induced by fixed shadows, the outer spirals are leading, and the rotational direction of the disk is clockwise. Such a difference in the gas kinematics will likely be tested by forthcoming ALMA observations of HD 100453.

\subsection{Shadows-induced scale height variations}

At a given radius while orbiting the star, the gas periodically goes from an illuminated region, with large irradiation, to one with negligible irradiation heating (the shadow). Assuming that the cooling and heating timescales are shorter than the dynamical (orbital) timescale, the gas temperature and the pressure are lower in these shadowed regions. As the pressure support of the gas fails, the gas falls towards the midplane, reducing the scale height. Upon exiting the shadow, the gas is heated again, causing the column to expand vertically again. This modulation of the disk scale height might affect the appearance of the rim in scattered light. To quantify this effect, we consider a single radius of the rim that is directly illuminated by the star. At this radius, the temperature contrast is the strongest between the rim and the shadowed regions, and we assume that radii in the far reaches of the shadow that receive grazing radiation can be neglected. We applied Newton's second law of motion to $H$, the pressure scale height. We consider the vertical hydrostatic balance equation in the disk as a starting point and follow the evolution of a vertical gas parcel along the rim as:

$$
\frac{\mathrm{d}^{2} H(t)}{\mathrm{d} t^{2}}=-\underbrace{\Omega_{\mathrm{K}}^{2} H(t)}_{1}+\underbrace{\frac{c_{\mathrm{s}}(t)^{2}}{H(t)}}_{2}-\underbrace{\Gamma \frac{\mathrm{d} H(t)}{\mathrm{d} t}}_{3},
$$

where $c_{\mathrm{s}}$ is the sound speed, $\Omega_{\mathrm{K}}$ the orbital Keplerian frequency, and $\Gamma$ a damping factor. This second order equation is similar to that of a driven damped oscillator. On the right hand side of Eq. (5), (1) describes the vertical component of the gravitational force that tries to contract the disk; (2) is the vertical pressure force that intends to expand the disk and (3) is a damping term, that mimics the loss of energy. $\Gamma$ is used to characterize the strength of the damping force and is assumed to be on the order of the dynamical time scale $1 / \Omega_{\mathrm{K}}$. For simplicity, we assume instant cooling and heating, so we take the sound speed to be a step function, and choose $c_{\mathrm{s}, \min } / c_{\mathrm{s}, \max }=0.6$, as computed from the temperature in the shadows in our best radiative transfer model.

Figure 7 shows the assumed sound speed profile and the modeled disk scale height for a single orbital period (i.e., two periods in the oscillation because of the two shadows). Just before entering the shadow, the disk scale height reaches a peak height and increases above the initial value, due to the inertia of the material. A variation in scale height changes the amount of stellar radiation intercepted by the disk and, at these locations, the rim scatters more stellar light and appears brighter. The width of this brightened region is related to the sound speed variation inside and outside of the shadows, and to the damping parameter. This leads to an asymmetric brightness distribution along the rim, the amplitude of which is determined by the pressure difference between shadowed and illuminated regions. Note that in Fig. 7, the disk scale height is plotted against the azimuthal angle $\phi=\Omega_{\mathrm{K}} t$, which increases in the clockwise direction to match the observed locations of the bright regions along the rim. To approximately estimate the effect on the scattered light brightness, we assume that the brightness varies proportionally to the scale height, and multiply the scale height by the incoming radiation of the star, neglecting the effects of inclination and scattering angle. We find a maximum amplitude of $20 \%$ brightness variation along the rim. In the extreme case of $c_{\mathrm{s}, \min } / c_{\mathrm{s}, \max }=0$, the maximum amplitude reaches $40 \%$, still significantly less than the factor 2 observed (see Fig. 4; between PAs of $125^{\circ}$ and $270^{\circ}$, and PAs of $320^{\circ}$ and $60^{\circ}$ ).

In contrast, as shown in Sect. 4, our radiative transfer model produces an azimuthally asymmetric brightness distribution that matches the observations well. This is due to the polarization efficiency being maximal along the semi-major axis. This effect likely dominates, and can be amplified by the scale height variations along the rim, in particular on the far side of the (inclined) disk, for which we directly see the rim front. However, these scenarios cannot be disentangled because, by chance, the shadows are located close to the major axis.

\subsection{Origin of a misaligned inner disk}

Shadows have now been detected in a handful of disks (Stolker et al. 2016a; Pinilla et al. 2015b; Canovas et al. 2016; Avenhaus et al. 2014). A strongly misaligned inner disk is assumed to explain the presence of two shadows (Marino et al. 2015), but the origin of such a misalignment is an open question.

A massive planetary- or low stellar-mass companion that would carve a dust cavity inside $20 \mathrm{au}$, and on an inclined orbit with respect to the outer disk, could possibly lead to a misaligned inner disk. Such a companion was detected in the cavity of the disk HD 142527 (Biller et al. 2012; Close et al. 2014) and found to be on an eccentric orbit (Lacour et al. 2016). If the outer disk holds a significant amount of gas, it is not clear how long such a misalignment can be sustained. Depending on the location and mass of the companion, the linear theory predicts that it can last $\sim 1$ Myr at most (Foucart \& Lai 2013). However, if the inner disk 


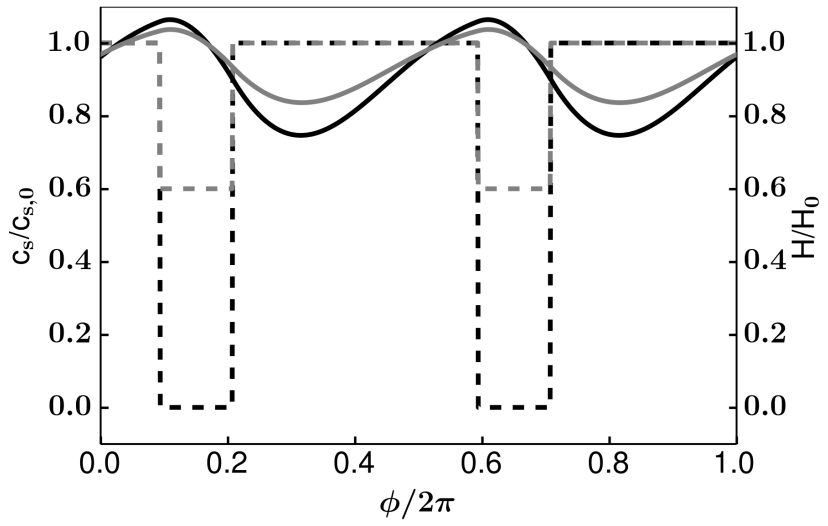

Fig. 7. Isothermal sound speed profile (dashed) and scale height of the disk as a function of azimuthal angle along the ring (solid) in two cases where $c_{\mathrm{s}, \min } / c_{\mathrm{s}, \max }=0.6$ (gray) and $c_{\mathrm{s}, \min } / c_{\mathrm{s}, \max }=0$ (black). All quantities are normalized. Note that the co-moving time increases towards the right. We set $H(t=0)=H_{0}=1.0$.

is highly misaligned, the timescale can be much longer due to the Kozai mechanism, an inclination/eccentricity pumping effect. If it is also on an inclined orbit, the M-dwarf companion could, in turn, influence the inner companion's orbit (Lubow \& Martin 2016; Martin et al. 2016; Casassus et al. 2015).

A massive companion inside the cavity could also explain the low gas-to-dust ratio and the very low mass accretion rate, estimated for this object (Collins et al. 2009). The inner companion would halt material from flowing closer in towards the star, which would lead to an inner disk resembling a debris disk belt inside $1 \mathrm{au}$. This inner belt should still be radially optically thick enough to cast two shadows on the rim, whilst having a scale height substantial enough to strongly reprocess light in the NIR regime. Dust at large scale height could be due to dynamical scattering of dust grains by the inner companion (Krijt \& Dominik 2011).

\section{Conclusions}

In this paper, we present polarized scattered light optical and NIR images of the $10 \mathrm{Myr}$ protoplanetary disk around the Herbig Ae star HD 100453, obtained with VLT/SPHERE. We report on the detection of a ring like feature, two spiral arms, and two shadows located very close to the spirals. We also detect a faint spiral like feature in the SW.

We present a radiative transfer model that efficiently accounts for the main characteristics of these features, and discuss the hydrodynamical consequences of the change in stellar irradiation at the shadows' locations. We find that:

1. the properties of the shadows (location, width, contrast) are well reproduced using an inner and an outer disk misaligned by $72^{\circ}$. Their morphology depends on the inner disk aspect ratio, and on the width and shape of the outer disk rim;

2. the faint spiral-like feature detected in the SW could trace the scattering surface of the bottom side of the disk, if the disk is tidally truncated by the M-dwarf companion currently seen at a projected distance of $119 \mathrm{au}$;

3. the strong azimuthal brightness variations observed along the rim can be well reproduced by the scattering phase function using small dust grains up to $1 \mu \mathrm{m}$ in size;

4. the local changes in stellar irradiation induces a modulation in the disk scale height that may amplify this effect.
The origin of the spirals, however, remains unclear. While the M-dwarf companion can produce the observed $m=2$ mode (Dong et al. 2016), the clear connection of the spirals with the shadows is puzzling, and if not coincidental, means that the shadows may also play a role in triggering the spirals (Montesinos et al. 2016). Another open question is how a $72^{\circ}$ misalignment between the inner and outer disk can be generated, and whether this points towards the presence of an additional, yet undetected, massive companion inside the cavity.

ALMA observations of this disk will undoubtedly shed light on many of these questions. It will not only be possible to estimate the gas and dust mass in the cavity and outer disk with more sensitive observations than the ones available today, but also to measure the kinematics of the gas. This may constrain the presence of a massive companion therein (Perez et al. 2015), and will indicate whether the spirals are leading or trailing, possibly constraining their formation mechanism. These observations will also accurately constrain the outer edge of the disk, which will then show whether the faint feature located in the SW is indeed the bottom side of a truncated disk, or is, in fact, another spiral arm.

Acknowledgements. We acknowledge the team at Paranal for their help during the observations and the referee for his/her suggestions. M.B. wishes to thank J.-P. Berger, M. Flock, L. Klarmann, and B. Lazareff for fruitful discussions. SPHERE is an instrument designed and built by a consortium consisting of IPAG (Grenoble, France), MPIA (Heidelberg, Germany), LAM (Marseille, France), LESIA (Paris, France), Laboratoire Lagrange (Nice, France), INAF-Osservatorio di Padova (Italy), Observatoire de Genève (Switzerland) ETH Zurich (Switzerland), NOVA (Netherlands), ONERA (France) and ASTRON (Netherlands) in collaboration with ESO. SPHERE was funded by ESO, with additional contributions from CNRS (France), MPIA (Germany), INAF (Italy), FINES (Switzerland) and NOVA (Netherlands). SPHERE also received funding from the European Commission Sixth and Seventh Framework Programmes as part of the Optical Infrared Coordination Network for Astronomy (OPTICON) under grant number RII3-Ct-2004-001566 for FP6 (20042008), grant number 226604 for FP7 (2009-2012) and grant number 312430 for FP7 (2013-2016). We acknowledge financial support from the Programme National de Planétologie (PNP) and the Programme National de Physique Stellaire (PNPS) of CNRS-INSU. This work has also been supported by a grant from the French Labex OSUG@2020 (Investissements d'avenir, ANR10 LABX56). This work has made use of the SPHERE Data Centre, jointly operated by OSUG/IPAG (Grenoble), PYTHEAS/LAM (Marseille), OCA/Lagrange (Nice) and Observatoire de Paris/LESIA (Paris). The results reported herein benefitted from collaborations and/or information exchange within NASA's Nexus for Exoplanet System Science (NExSS) research coordination network sponsored by NASA's Science Mission Directorate. A.J. acknowledges the support by the DISCSIM project, grant agreement 341137 funded by the European Research Council under ERC-2013-ADG. A.Z., S.D, R.G. D.M., E.S. acknowledge support from the "Progetti Premiali" funding scheme of the Italian Ministry of Education, University, and Research.

\section{References}

ALMA Partnership, Brogan, C. L., Pérez, L. M., et al. 2015, ApJ, 808, L3 Andrews, S. M., Wilner, D. J., Zhu, Z., et al. 2016, ApJ, 820, L40

Apai, D., Pascucci, I., Brandner, W., et al. 2004, A\&A, 415, 671

Apai, D., Kasper, M., Skemer, A., et al. 2016, ApJ, 820, 40 Ardila, D. R., Golimowski, D. A., Krist, J. E., et al. 2007, ApJ, 665, 512 Avenhaus, H., Quanz, S. P., Schmid, H. M., et al. 2014, ApJ, 781, 87 Benisty, M., Natta, A., Isella, A., et al. 2010, A\&A, 511, A74 Benisty, M., Juhasz, A., Boccaletti, A., et al. 2015, A\&A, 578, L6 Béthune, W., Lesur, G., \& Ferreira, J. 2016, A\&A, 589, A87 Beuzit, J.-L., Feldt, M., Dohlen, K., et al. 2008, SPIE Proc., 7014, 18 Biller, B., Lacour, S., Juhász, A., et al. 2012, ApJ, 753, L38 Boccaletti, A., Abe, L., Baudrand, J., et al. 2008, Adaptive Optics Systems, Proc. SPIE, 7015, 70151B

Brittain, S. D., Simon, T., Najita, J. R., \& Rettig, T. W. 2007, ApJ, 659, 685

Canovas, H., Ménard, F., Hales, A., et al. 2013, A\&A, 556, A123

Canovas, H., Ménard, F., de Boer, J., et al. 2015, A\&A, 582, L7

Canovas, H., Hardy, A., Zurlo, A., et al. 2016, A\&A, in press, DOI: $10.1051 / 0004-6361 / 201629145$

Carrasco-González, C., Henning, T., Chandler, C. J., et al. 2016, ApJ, 821, L16 
Casassus, S. 2016, PASA, 33, e013

Casassus, S., Perez M., S., Jordán, A., et al. 2012, ApJ, 754, L31

Casassus, S., Marino, S., Pérez, S., et al. 2015, ApJ, 811, 92

Chen, X. P., Henning, T., van Boekel, R., \& Grady, C. A. 2006, A\&A, 445, 331

Christiaens, V., Casassus, S., Perez, S., van der Plas, G., \& Ménard, F. 2014, ApJ, 785, L12

Claudi, R. U., Turatto, M., Gratton, R. G., et al. 2008, in Ground-based and Airborne Instrumentation for Astronomy II, Proc. SPIE, 7014, 70143E

Close, L. M., Follette, K. B., Males, J. R., et al. 2014, ApJ, 781, L30

Collins, K. A., Grady, C. A., Hamaguchi, K., et al. 2009, ApJ, 697, 557

de Boer, J., Salter, G., Benisty, M., et al. 2016, A\&A, 595, A114

Dipierro, G., Pinilla, P., Lodato, G., \& Testi, L. 2015, MNRAS, 451, 974

Dohlen, K., Langlois, M., Saisse, M., et al. 2008, SPIE Proc., 7014, 10

Dominik, C., Dullemond, C. P., Waters, L. B. F. M., \& Walch, S. 2003, A\&A, 398, 607

Dong, R., Zhu, Z., Fung, J., et al. 2016, ApJ, 816, L12

Fairlamb, J. R., Oudmaijer, R. D., Mendigutía, I., Ilee, J. D., \& van den Ancker, M. E. 2015, MNRAS, 453, 976

Flock, M., Ruge, J. P., Dzyurkevich, N., et al. 2015, A\&A, 574, A68

Flock, M., Fromang, S., Turner, N. J., \& Benisty, M. 2016, ApJ, 827, 144

Foucart, F., \& Lai, D. 2013, ApJ, 764, 106

Fusco, T., Rousset, G., Sauvage, J.-F., et al. 2006, Opt. Express, 14, 7515

Garufi, A., Quanz, S. P., Avenhaus, H., et al. 2013, A\&A, 560, A105

Garufi, A., Quanz, S. P., Schmid, H. M., et al. 2016, A\&A, 588, A8

Ginski, C., Stolker, T., Pinilla, P., et al. 2016, A\&A, 595, A112

Grady, C. A., Muto, T., Hashimoto, J., et al. 2013, ApJ, 762, 48

Habart, E., Natta, A., Testi, L., \& Carbillet, M. 2006, A\&A, 449, 1067

Hanson, J. R., \& Apai, D. 2015, Am. Astron. Soc. Meet. Abstr., 225, 258.13

Hashimoto, J., Tamura, M., Muto, T., et al. 2011, ApJ, 729, L17

Juhász, A., Benisty, M., Pohl, A., et al. 2015, MNRAS, 451, 1147

Klarmann, L., Benisty, M., Min, M., et al. 2016, A\&A, submitted

Khalafinejad, S., Maaskant, K. M., Mariñas, N., \& Tielens, A. G. G. M. 2016, A\&A, 587, A62

Kouwenhoven, M. B. N., Brown, A. G. A., Zinnecker, H., Kaper, L., \& Portegies Zwart, S. F. 2005, A\&A, 430, 137

Krijt, S., \& Dominik, C. 2011, A\&A, 531, A80

Kuhn, J. R., Potter, D., \& Parise, B. 2001, ApJ, 553, L189

Lacour, S., Biller, B., Cheetham, A., et al. 2016, A\&A, 590, A90

Langlois, M., Vigan, A., Moutou, C., et al. 2013, in Proc. Third AO4ELT Conference, eds. S. Esposito, \& L. Fini, 63

Langlois, M., Dohlen, K., Vigan, A., et al. 2014, SPIE Proc., 9147, 1

Lazareff, B., Berger, J.-P., Kluska, J., et al. 2017, in press, DOI: $10.1051 / 0004-6361 / 201629305$

Lodato, G., \& Rice, W. K. M. 2004, MNRAS, 351, 630

Lubow, S. H., \& Martin, R. G. 2016, ApJ, 817, 30

Lyra, W., Turner, N. J., \& McNally, C. P. 2015, A\&A, 574, A10

Maire, A.-L., Langlois, M., Dohlen, K., et al. 2016, Ground-based and Airborne Instrumentation for Astronomy VI, Proc. SPIE, 9908, 990834

Marino, S., Perez, S., \& Casassus, S. 2015, ApJ, 798, L44

Martin, R. G., Lubow, S. H., Nixon, C., \& Armitage, P. J. 2016, MNRAS, 458, 4345

Meeus, G., Waters, L. B. F. M., Bouwman, J., et al. 2001, A\&A, 365, 476

Menu, J., van Boekel, R., Henning, T., et al. 2015, A\&A, 581, A107

Min, M., Hovenier, J. W., \& de Koter, A. 2005, A\&A, 432, 909

Min, M., Dullemond, C. P., Dominik, C., de Koter, A., \& Hovenier, J. W. 2009, A\&A, 497, 155

Montesinos, M., Perez, S., Casassus, S., et al. 2016, ApJ, 823, L8

Muto, T., Grady, C. A., Hashimoto, J., et al. 2012, ApJ, 748, L22

Ogilvie, G. I., \& Lubow, S. H. 2002, MNRAS, 330, 950

Papaloizou, J. C. B., \& Terquem, C. 1995, MNRAS, 274, 987

Pavlov, A., Möller-Nilsson, O., Feldt, M., et al. 2008, Advanced Software and Control for Astronomy II, Proc. SPIE, 7019, 701939

Pérez, L. M., Isella, A., Carpenter, J. M., \& Chandler, C. J. 2014, ApJ, 783, L13

Pérez, L. M., Carpenter, J. M., Andrews, S. M., et al. 2016, Science, 353, 1519

Perez, S., Dunhill, A., Casassus, S., et al. 2015, ApJ, 811, L5

Petit, C., Sauvage, J.-F., Fusco, T., et al. 2014, SPIE Proc., 9148

Pinilla, P., de Boer, J., Benisty, M., et al. 2015a, A\&A, 584, L4

Pinilla, P., de Juan Ovelar, M., Ataiee, S., et al. 2015b, A\&A, 573, A9

Pinilla, P., van der Marel, N., Pérez, L. M., et al. 2015c, A\&A, 584, A16

Pinte, C., Dent, W. R. F., Ménard, F., et al. 2016, ApJ, 816, 25

Pohl, A., Pinilla, P., Benisty, M., et al. 2015, MNRAS, 453, 1768

Quanz, S. P., Schmid, H. M., Geissler, K., et al. 2011, ApJ, 738, 23

Rapson, V. A., Kastner, J. H., Millar-Blanchaer, M. A., \& Dong, R. 2015, ApJ, 815, L26
Roelfsema, R., Schmid, H. M., Pragt, J., et al. 2010, SPIE Proc., 7735, 4

Ruge, J. P., Flock, M., Wolf, S., et al. 2016, A\&A, 590, A17

Sauvage, J., Fusco, T., Petit, C., et al. 2014, SPIE Proc., 9148

Schmid, H. M., Joos, F., \& Tschan, D. 2006, A\&A, 452, 657

Soummer, R., Pueyo, L., \& Larkin, J. 2012, ApJ, 755, L28

Stolker, T., Dominik, C., Avenhaus, H., et al. 2016a, A\&A, 595, A113

Stolker, T., Dominik, C., Min, M., et al. 2016b, A\&A, 596, A70

Tang, Y.-W., Guilloteau, S., Piétu, V., et al. 2012, A\&A, 547, A84

Thalmann, C., Schmid, H. M., Boccaletti, A., et al. 2008, in Ground-based and

Airborne Instrumentation for Astronomy II, Proc. SPIE, 7014, 70143F

Tsukagoshi, T., Nomura, H., Muto, T., et al. 2016, ApJ, 829, L35

van der Marel, N., Pinilla, P., Tobin, J., et al. 2015, ApJ, 810, L7

Verhoeff, A. P., Min, M., Pantin, E., et al. 2011, A\&A, 528, A91

Vigan, A., Moutou, C., Langlois, M., et al. 2010, MNRAS, 407, 71

Wagner, K., Apai, D., Kasper, M., \& Robberto, M. 2015a, ApJ, 813, L2

Wagner, K. R., Sitko, M. L., Grady, C. A., et al. 2015b, ApJ, 798, 94

Wagner, K., Apai, D., Kasper, M., et al. 2016, Science, 353, 673

Woitke, P., Min, M., Pinte, C., et al. 2016, A\&A, 586, A103

Wolff, S. G., Perrin, M., Millar-Blanchaer, M. A., et al. 2016, ApJ, 818, L15

1 Univ. Grenoble Alpes, CNRS, IPAG, 38000 Grenoble, France e-mail: Myriam. Benisty@univ-grenoble-alpes . fr

2 Anton Pannekoek Institute for Astronomy, University of Amsterdam, Science Park 904, 1098 XH Amsterdam, The Netherlands

3 Max Planck Institute for Astronomy, Königstuhl 17, 69117 Heidelberg, Germany

4 Leiden Observatory, Leiden University, PO Box 9513, 2300 RA Leiden, The Netherlands

5 Institute for Theoretical Astrophysics, Heidelberg University, Albert-Ueberle-Strasse 2, 69120 Heidelberg, Germany

6 CRAL, UMR 5574, CNRS, Université Lyon 1, 9 avenue Charles André, 69561 Saint-Genis-Laval Cedex, France

7 SRON Netherlands Institute for Space Research, Sorbonnelaan 2, 3584 CA Utrecht, The Netherlands

8 Department of Astronomy/Steward Observatory, The University of Arizona, 933 North Cherry Avenue, Tucson, AZ 85721, USA

9 Institute of Astronomy, Madingley Road, Cambridge CB3 OHA, UK

10 Max-Planck-Institut fur Extraterrestrische Physik, Giessenbachstrasse 1, 85748 Garching, Germany

11 Universidad Autonónoma de Madrid, Dpto. Física Teórica, Facultad de Ciencias, Campus de Cantoblanco, 28049 Madrid, Spain

12 Institute for Astronomy, ETH Zurich, Wolfgang-Pauli-Strasse 27, 8093 Zurich, Switzerland

13 Núcleo de Astronomía, Facultad de Ingeniería, Universidad Diego Portales, Av. Ejercito 441, Santiago, Chile

14 Departamento de Astronomía, Universidad de Chile, Casilla 36-D, 1058 Santiago, Chile

15 LESIA, Observatoire de Paris-Meudon, CNRS, Université Pierre et Marie Curie, Université Paris Didierot, 5 place Jules Janssen, 92195 Meudon, France

16 INAF-Osservatorio Astronomico di Padova, Vicolo dell'Osservatorio 5, 35122 Padova, Italy

17 Institute for Astronomy, University of Edinburgh, Blackford Hill View, Edinburgh EH9 3HJ, UK

18 European Southern Observatory, Karl-Schwarzschild-Str. 2, 85748 Garching, Germany

19 Aix-Marseille Univ, CNRS, LAM, Laboratoire d'Astrophysique de Marseille, 13013 Marseille, France

20 INAF-Osservatorio Astrofisico di Arcetri, Largo E. Fermi 5, 50125 Firenze, Italy

21 ONERA - 29 avenue de la Division Leclerc, 92322 Chatillon Cedex, France

22 Geneva Observatory, Univ. of Geneva, Chemin des Maillettes 51, 1290 Versoix, Switzerland 


\section{Appendix A: Angular differential imaging}

In this section we present angular differential imaging (ADI) images obtained with SPHERE in 2015 and in 2016.

We reprocessed the 2015 data in the ESO archive that were published in Wagner et al. (2015a). In the aforementioned discovery paper, reference differential imaging was used to investigate the inner structures of the disk $\left(0.15-0.4^{\prime \prime}\right)$. This method outperforms ADI at the innermost radii (where large field rotation is required for efficient ADI), but changing conditions throughout the observations led to differences in the PSF of the reference star and science target and thus shallower than needed contrast to detect the fainter outer disk features. To recover these features in the 2015 data, we performed a second independent angular differential imaging reduction of these data, in which the intrinsic field rotation of the Alt-Az telescope is utilized to model the stellar PSF separately from the other astrophysical sources in the image. We post-processed the SPHERE-IFS data through analysis and subtraction of the principal components of the PSF via the KLIP method (Soummer et al. 2012) using selfdeveloped IDL routines (Hanson \& Apai 2015; Apai et al. 2016; Wagner et al. 2016). In modeling and subtracting the PSF from each science frame we rejected frames in which the field had rotated by less than $1.5 \times F W H M$ pixel separation to avoid selfsubtraction of the disk structures. Over the course of the observations the field rotated by $12.5^{\circ}$, allowing us to investigate the regions beyond $0.4^{\prime \prime}$ in high-contrast. The result is the detection at $Y, J$, and $H$-bands of the same faint third arm-like feature identified in the polarized intensity images, yielding confidence in its astrophysical nature.

In addition, HD 100453 was observed on January 20th, 2016, as part of the SHINE survey for Guaranteed Time Observation (GTO), using the Dual Band Imaging mode (DBI; Vigan et al. 2010) of the IRDIS instrument, with dual band filters $H 2$ and $H 3$ simultaneously. In parallel, a data cube was obtained with the near-IR Integral Field Spectrograph (IFS; Claudi et al. 2008) in $Y J$ mode. These observations were obtained with the Apodized Lyot Coronagraph (mask diameter: 185 mas, Boccaletti et al. 2008). We obtained a sequence of $4000 \mathrm{~s}$ in total on both instruments with a field rotation of $30 \mathrm{deg}$. Non-coronagraphic frames were obtained before and after the coronagraphic sequence for photometric calibration. Conditions were rather medium (seeing $\sim 1.1^{\prime \prime}$ ). The field orientation of IRDIS and IFS are derived from astrometric calibrations as described in Maire et al. (2016). All the data were reduced with the SPHERE pipeline (Pavlov et al. 2008) implemented at the SPHERE Data Center together with additional tools developed for the handling GTO data reduction. This includes dark and sky subtraction, bad-pixels removal, flat-field correction, anamorphism correction (Maire et al. 2016), and wavelength calibration for IFS. The
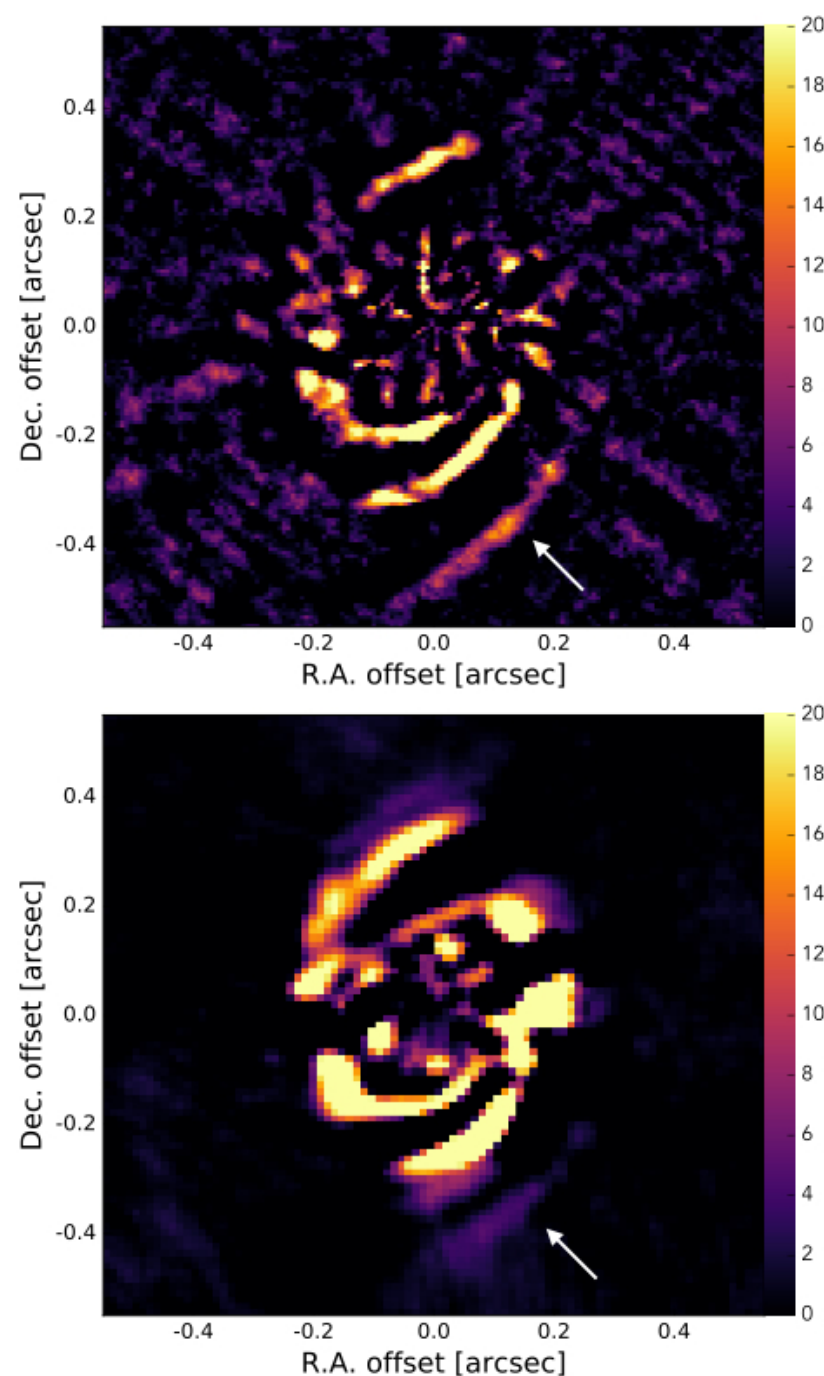

Fig. A.1. ADI images from 2015 (top, Wagner et al. 2015a) and from 2016 (bottom). The right arrows indicate the location of the faint third spiral-like feature that we interpret as the outer edge of the scattering surface on the bottom side of the disk.

location of the star is identified using the four symmetrical satellite spots generated by diffraction from a periodic waffle pattern introduced by an appropriate modification of the adaptive optics reference slopes sent by the deformable mirror (Langlois et al. 2013). Then, to remove the stellar halo and to achieve high contrast, the data were processed with the GTO high-level processing pipeline: SpeCal, which was developed for the SPHERE survey (R. Galicher, priv. comm.). 
M. Benisty et al.: Shadows and spirals in the protoplanetary disk HD 100453

\section{Appendix B: RT modeling}
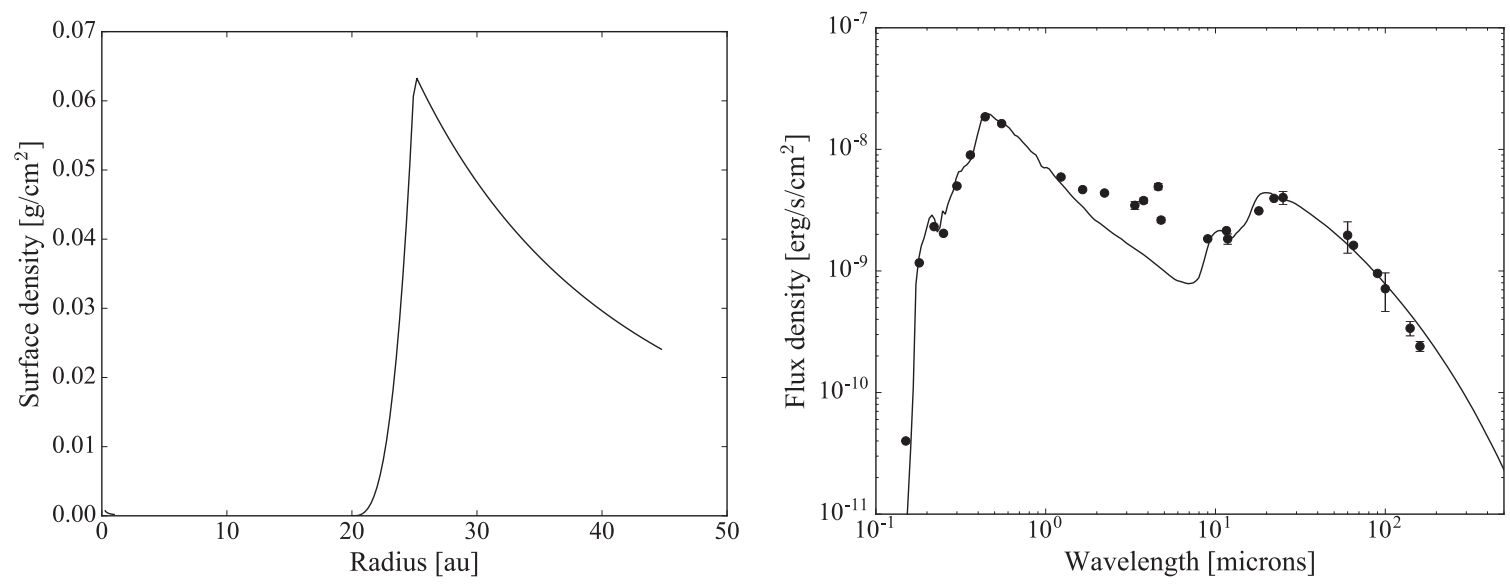

Fig. B.1. Left: surface density used in our radiative transfer model. Right: modeled SED compared to the observed photometry (from Khalafinejad et al. 2016). 